\title{
CONCIENCIA TECNOBUROCRATICA EN LOS GRUPOS SOCIALES DIRIGENTES. EL CASO DE CATALUNYA
}

\author{
Raúl García Durán \\ (Facultad de Económicas \\ de la Universitat Autonoma de Barcelona)
}

Ya desde los primeros años del poder bolchevique, el pensamiento social elaboró el concepto de la formación de una nueva clase social, distinta de la burguesía y el proletariado, que tendía a ser dominante ante la descomposición de la propiedad burguesa. Rizzi y Burnham fueron los primetos en señalar que no era preciso que dicha descomposición fuera violenta y que también en Occidente, en el seno del capital, se iba consolidando la nueva clase a través del poder gerencial. ${ }^{2}$ Es curioso resaltar cómo el concepto es de origen «izquierdista», elaborado por el pensamiento anarquista, las fracciones más radicales del partido bolchevique y dentro del trostkismo, y sin embargo en la actualidad es rechazado, salvo excepciones individuales, por este tipo de planteamientos como legitimación del poder gerencial precisamente. Soy una de esas excepciones individuales, creo que el concepto de la nueva clase es el que mejor nos explica el momento histórico en que nos encontramos y he intentado, en mi tesis doctoral, empezar a mostrarlo. La tesis se compone de 4 partes claramente delimitadas:

1. La historia del concepto, el por qué de ese techazo, básicamente: a) su origen en el análisis de lo que ocurte en la URSS y reducción de su

1. B. Rizzi, La burocratización del mundo, Península, 1980; J. Burnham, La revolución de los directores, Huemul, 1962.

2. R. García-Durán, «Un modelo histórico de definición de una nueva clase, la tecnobutocracia profesional. Inicio de su estudio empírico en Catalunya», UAB, 1977. 
contenido a crítica antiburocrática; $b$ ) su formulación como fin del capitalismo («revolución» de los gerentes) y utilización legitimadora (sociedad postindustrial técnica e inevitable); y $c$ ) el economicismo-mecanicismo dominante en el materialismo histórico: o capitalismo o socialismo y no bay ninguna otra posibilidad. ${ }^{3}$

2. La revisión del materialismo histórico que nos permite comprender la formación de la nueva clase a partir de la concepción de la historia como historicidad (reivindicación del método histórico) y de la superación de la determinación por «lo económico».

3. El proceso histórico de formación de la nueva clase. El enfrentamiento burguesía-proletariado va desarrollando determinados grupos sociales profesionales, en concreto la jerarquía científico técnica, la burocracia política, el poder gerencial, la burocracia obrera y un ejétcito cada vez más profesionalizado, en los que la burguesía, de origen tevolucionaria, delega tres fuentes de poder arrebatadas a la anterior aristocracia dominante: las armas, el ejercicio del poder político y el conocimiento. En definitiva, que mientras la burguesía se define por la propiedad de los medios de producción, otta propiedad, la del conocimiento, dividida en diferentes conocimientos especializados, queda en manos de grupos asalariados de profesionales de dichos conocimientos, al mantenerse en ellos la división del trabajo por oficios y no por tareas. Las modificaciones adoptadas por el capital ante la presión obrera hacen cada vez más crucial dicha propiedad del conocimiento (por delante de la propiedad meramente económica) generando las condiciones objetivas de fusión de dichos grupos en una fuerza social portadora de un modelo de organización social distinto al de la burguesía y al del proletariado.

4. La situación de la nueva clase es, sin embargo, aún de mayor consolidación en sí (intereses históricos objetivos específicos) que no para sí (consolidación como fuerza social), dependiendo en gran parte su futuro de dicha consolidación. El estudio de la nueva clase pasa así, en gtan parte hoy, por el análisis de hasta qué punto existe un modelo social común propio de la nueva clase y hasta qué punto se tiene conciencia de él entre los grupos sociales posibles apoyos objetivos de aquélla. Estudio que sólo puede ser concreto y que he centrado, he empezado a estudiar en Catalunya.

En este artículo pretendo recoger un breve resumen de esta última cuarta parte, del inicio del análisis empírico. Con todas las limitaciones

3. Una pequeña historia dei concepto puede encontrarse en el prólogo de S. Giner al texto de Rizzi ya citado; A. Castaños, ¿Tiene el socialismo su prebistoria?, Blume, 1977; P. Naville, Le nouveau Leviathan, Antrophos, 1969; M. Paillet, Marx contra Marx, Dopesa, 1971; y la presentación de Editorial Fontamara a su edición de L. Trotski, En defensa del marxismo, 1978. 
propias de un artículo y sobre un estudio incipiente, pero creo que el trabajo realizado proporciona una interesante visión de nuestros grupos sociales dirigentes (burguesía tradicional excluida) que vale la pena divulgar.

\section{DESCRIPCION DEL ESTUDIO REALIZADO}

Posibiemente, el estudio que más problemas metodológicos plantea es el intentar profundizar en una ideología. No creo que haya un método o una técrnica de investigación suficientemente rigurosa para elio y, quiero dejarlo claro ya de entrada, lo máximo que podemos obtener son aproximaciones. Si somos conscientes, en este sentido, de sus limitaciones (imagen que quiete dat el entrevistado, planteamientos inconscientes, etc.), parece que la entrevista es una técnica adecuada. $O$ al menos fue la técnica utilizada. Se trataba de hacer una serie de entrevistas a posibles «tecnoburócratas» representativos, en Catalunya, de las diferentes fracciones que, de acuerdo con la parte teórica, constituyen el origen de la nueva clase. La forma de selección de los entrevistados fue el buscar instituciones que podían ser consideradas representativas de los grupos sociales que forman dichas fracciones, excluyendo, por razones fáciles de entender (no ejército propio en Catalunya, incidencia aun del ejército franquista...), al ejército. Así:

- La representación más fácil, sin duda, era la de la burocracia política: se trataba de seleccionar altos cargos de la Generalitat, cargos políticos excluidos, distribuidos entre los diferentes departamentos y guardando la debida proporción entre funcionatios nuevos y antiguos transferidos. Pude contar con un buen informador en cada uno de los departamentos quedando al final la muestra compuesta por: 25 jefes de servicio, 7 ditectores generales, 5 jefes de gabinete técnico, 1 jefe de sección, 1 conseller (su personalidad lo aconsejaba) y un secretario general. En total 40 entrevistas. El número 40 es puramente pragmático; con él conseguí la mínima representación buscada en cada departamento ${ }_{2}^{4}$ aplicándolo después a cada una de las diferentes fracciones para facilitar la comparación cuantitativa. Un total de 160 entrevistas parecía un número lo suficientemente rico al tiempo que una tarea, aunque ardua, posible.

- La burocracia obrera también fue de fácil selección. Se puede discutir si estos partidos son burocráticos o no, pero está claro cuáles son los

4. Excluido el de Sanidad, fue imposible conectar con el informador previsto. 
partidos con más incidencia. Podía habet optado por una mayor profundización en la burocracia sindical, pero se trataba un poco de escoger y ya procuré en cada uno de los partidos entrevistar a un número suffciente de cargos sindicales. La distribución entre partidos fue puramente cuantitativa: 20 entrevistas en el PSUC (miembros del C. Central) y 20 en el PSC (Consell Nacional).

- Para la representación del poder gerencial seleccioné el Círculo de Economía, institución que se ha destacado suficientemente en la modernización de nuestra estructura empresarial. Conseguí en el Círculo 22 entrevistas (18 miembros de la Junta y 4 socios especialmente activos miem. bros de juntas anteriores), de forma que decióí completarlas con 11 entre. vistas en ESADE (muestra de los diferentes departamentos y del Patrona. to) y 7 con directivos de una importante empresa multinacional de fuerte implantación en Catalunya.

- En cuanto a la jerarquía técnica, mis intenciones fueron 20 entrevistas en la Junta Directiva del Colegio de Economistas y 20 en los de Ingenieros (10 en Industriales y 10 en Caminos). Ya muy avanzado el trabajo, me fue completamente imposible realizar 4 de dichas entrevistas ( 2 en el Colegio de Economistas y 2 en el de Ing. Industriales), que sustituí por técnicos de empresas conectadas a través del Círculo. Así, incluso se compensaba en parte la excesiva representación de los profesionales libres que se puede encontrar en las juntas de los Colegios.

Las entrevistas fueron abiertas, pero siguiendo un cuestionario común -que recojo en Anexo- de forma que fuera posible la comparación. Com* paración que realicé a dos niveles: análisis cualitativo, recogiendo las respuestas más significativas y seleccionando los 4 tipos ideológicos ideales que parecen detectarse entre los entrevistados, y análisis cuantitativo, cetrando las respuestas e institución por institución. Aquí nos tendremos que limitar, desgraciadamente, a las conclusiones, que quedarán así no con* trastadas, sólo enunciadas. Remito al lector al conjunto de la investigación. ${ }^{5}$

Pero antes de empezar quizá sea bueno tesumir las respuestas a las 4 primeras preguntas, que nos acaban de defnir, en concreto, la «muestra» escogida:

- El intervalo de edad más común es entre 36 y 45 años, superior a los 30.34 que constinuye el intervalo donde la actividad es mayor en el conjunto de la población catalana: 6 la cartera profesional exige tiempo.

5. A tener en cuenta cuando se trate de interpretar los porcentajes cuantitativos.

6. A. Sáez, La actividad económica de los catalanes, 1974. 
- Se trata mayotitariamente de una población titulada superior $(88,8 \%)$, siendo sólo en los partidos donde este porcentaje es inferior al $90 \%: 55 \%$ en el PSUC y $80 \%$ en el PSC.

- La carrera habitual es, con mucho, Económicas, seguida de Ingeniería (aun teniendo en cuenta la distorsión que significa el estudio de los Colegios escogidos). Es muy elevado el número de abogados, pero en general como carrera complementaria, acompañando a otra, al igual que Dirección de Empresas, que parece sustituir al Derecho entre los más jóvenes.

- El currículum mayoritario es a través de los cargos directivos de la empresa, completado con dedicación parcial o coyuntural a la docencia universitaria y a los servicios de estudios. Sólo en los partidos es mayor el origen en esta segunda, y se observa un cierto ascenso desde trabajos administrativos.

- Por último, y teniendo en cuenta la distorsión que significan los partidos, que se votan lógicamente a sí mismos, nuestra muestra vota Convergència $\dot{i}$ Unió $(55,7 \%)$ por encima de los votos obtenidos en la última elección realizada antes de las entrevistas $(46,6 \%$ en 1984). El apoyo es principalmente fuerte en la tecnoestructura gerencial $(73,6 \%)$, superior incluso al de la Generalitat $(58,8 \%)$, lo cual parece confrimar lo que me decía uno de los entrevistados, la falsedad de la imagen de Convergencia como partido de los botiguers y su acercamiento precisamente a la tecnoburocracia. Entre los técnicos, el $52 \%$ vota PSC, confirmándose así una de las hipótesis mantenidas: el intento de expresión a través de la social. democracia de la jerarquía técnica no llegada a la cúspide.

La selección principal quedó completada con un grupo de contraste (10 entrevistas), formado por profesionales de un centro de estudios y una asesoría laboral, en busca de profesionales que pudieran tener, sin embargo, una ideología muy distinta de la que se intentaba constatar. Las primeras preguntas confirmaron ya de entrada la validez del grupo: mismos datos objetivos (salvo un inferior currículum empresarial) y sin embargo el $90 \%$ habia optado por la abstención.

\section{RASGOS IDEOLOGICOS}

La conclusión principal de nuestro trabajo es la constatación de que realmente se puede hablar, aunque con el PSUC como frontera (véase supra), de una ideología común en el conjunto de la muestra, de una concepción de la dinámica social, un modelo de desartollo social, una caracterización de las clases, comunes. Veamos sus rasgos definitorios: 


\section{Concepción de la bistoria como progreso}

La respuesta a la pregunta 5 es explícita: el $93,2 \%$ concibe la histotia como una línea de progreso, y de ellos el $58,2 \%$ sin ningún reparo. Los reparos objetados por el resto serialan la existencia de un cierto resquicio entre el progreso material y el social. Es significativo que la casi totalidad sitúa la respuesta, el progreso, a partir de la Segunda Guerra Mundial, del actual modelo de desarrollo, cuna para mí de la nueva clase. Un ejemplo: «Es evidente, principalmente desde que se inventó la segu. ridad social. Ahora las crisis, por duras que sean, producen consecuencias mucho menos graves (...) aunque no se cubran al $100 \%$ las necesidades. $\mathrm{El}$ progreso es tanto material como social. Es el desarrollo industrial y tecnológico el que ha creado la clase media. Por otra parte, la aparición del ejecutivo no propietario es un avance importante (...) la empresa familiar ha dejado de existir, afortunadamente $y$, creo, en mejora de la misma empresa» (Círculo). Significativa respuesta prólogo-resumen al conjunto de planteamientos a considerar.

\section{Alta valoración, casi mitificación, del desarrollo científico-técnico como motor de dicho progreso}

Ello queda patente no sólo en las respuestas a la pregunta 12 , la más directa, y la 13, rechazo de los movimientos ecologistas en cuanto expresión de un modelo de desarrollo distinto, ${ }^{7}$ sino en la introducción por los en" trevistados del tema en la respuesta a muchas de las otras preguntas. De nuevo un ejemplo: «Enorme. Hay una frase que dijo hace un siglo, no me acuerdo quién, de que el motor de la historia es la lucha de clases. Hoy en dia mucha gente pensamos que el motor $(. .$.$) es el progreso científico-téc-$ nico. Las consecuencias sociales que se pueden derivar de los actuales avances tecnológicos y de los futuros (...) son importantísimas (...). El progreso científico-técnico sí que contribuye a la conciliación. Puede facilitar los instrumentos científicos y técnicos que hacen que la negociación sea lo más racional posible. Cuantos más argumentos técnicos se puedan aportar a la mesa de negociaciones, más grande puede ser la posibilidad de acuerdo" (Generalitat y Círculo). ${ }^{8}$

7. He tratado con más detalle el rechazo por nuestra muestra de los movimientos ecologistas en un artículo específico, «¿Qué opina la tecnoburocracia de los movimientos ecologistas?», Integral, pendiente de publicación.

8. Algún entrevistado constó en la muestra seleccionada de más de una institución. Los he contabilizado, a nivel cuantitativo, en cada una de ellas. 
El $60,6 \%$ de los entrevistados considera el progreso científico-técnico como determinante de la dinámica social, y el 64,3 lo consideta siempre positivo. Sólo un $7,5 \%$ habla de posibles consecuencias negativas (siempre parciales) y sólo 6 entrevistados se tefieren a la necesidad de una tecnología «blanda». ${ }^{9}$ En la pregunta 10 se constató que sólo el $1,9 \%$ cree posible un tecnológico «mundo feliz» huxleyano.

\section{Confianza en el crecimiento económico, pero racionalizado}

Tal como se fue concretando, la pregunta 7 nos mostró dicha confianza: predominio del crecimiento sobre otro tipo de orientación más ligada al bienestar, aunque fueron numerosas las respuestas en contra del crecimiento por el crecimiento: "hay que tener en cuenta el a costa de qué». A resaltar que las respuestas más orientadas hacia el «bienestar» concretaban éste básicamente a través de: a) satisfacción por todos de las necesidades básicas, $b$ ) igualdad de oportunidades, y c) aprovechamiento para el bienestar de la capacidad tecnológica alcanzada. Todo deja traslucir una otientación más meritocrática (a partir de unos mínimos) que igualitarista (véase supra).

La lógica económica es desde luego dominante, y ello se muestra en el $87,9 \%$ que cree en la posibilidad de una organización económica tacional, siendo lo más significativo el que sólo un $6,5 \%$ nos habla de distintas racionalidades posibles. Los No y los reparos a esa racionalidad vienen de las limitaciones de la ciencia económica. La racionalización es, desde luego, para la gran mayoría de entrevistados, fruto del saber: «Indudablemente, creo posible la organización económica de una forma racional. Indudablemente, la tienen que precisar los gabinetes de estudio, las personalidades y los que se dedican a pensar para los demás: es bueno qute así sea, pues ellos tienen los medios y una gran responsabilidad cientffica. Se tiene indudablemente que racionalizar, intervenir, aunque racionalizando también la intervención» (Generalitat).

No se trata, sin embargo, de la lógica económica al uso, ésta tiene que ser "racionalizada», la productividad es la clave del progreso económicosocial, pero matizada, no considerada sólo como productividad del trabajo sino a partir de:

9. A resaltar como nuestra muestra mantiene la idea de «neutralidad» de la ciencia salvo en la caractetización positiva de ésta. Un $14 \%$ señala que el efecto del progreso científico-técnico «depende de su uso», «depende de en qué manos esté», pero no hay ninguna respuesta explicitando que sus efectos están ya en la propia tecnología que teproduce las condiciones sociates en que ha sido creada. 
- El management: «De acuerdo siempre que por productividad se entienda el rendimiento tanto del de abajo como del de atriba. Normalmente se usa sólo respecto al trabajo. Es importante tener en cuenta la productividad del señor que organiza el trabajo, que es precisamente organizarlo bien» (Generalitat). «La productividad, además, no se puede considerar sólo en el sentido taylorista, sino que es general, y es básica la productividad de la administración y la dirección empresarial» (ESADE).

- La responsabilidad de cada uno en su sitio en la jerarquía: «El incremento de la productividad comporta un progreso, ahora bien, no es la palanca clave. Ésta es (...) el incremento de la responsabilidad por parte de todo el mundo» (Colegio de Ingenieros). "Si a la gente se le mentaliza sufscientetnente pata que encuentre interesante el trabajo que hace, la productividad viene por sí misma. En este sentido, sí que es importante la productividad, que la gente esté integrada en su faena, se sienta partícipe de ella, con una participación importante en lo que está haciendo» (Colegio de Ingenieros).

- El progreso científico-técnico: «La clave es el progreso científicotécnico que, evidentemente, llevará a su vez un incremento de la productividad» (Generalitat y Círculo). «La productividad es importante, pero más que ella el desarrollo tecnológico (...). Las industrias van pasando de intensivas en mano de obra a intensivas en capital. La investigación es tanto más importante que la productividad» (Ćrculo). «Ha habido épocas en que los incrementos de la productividad eran básicos económica y socialmente, fue cuando se inventaron todos los sistemas de productividad porque antes el trabajo no era ordenado, se hacían las cosas mal hechas, perdiendo tiempo. Por ello se inventó el aumentar el número de unidades por tiempo, el taylorismo fue una gran conquista intelectual (...). La productividad tiene sin embargo un límite. A una persona no se le puede pedir productividad más allá de un cietto punto: sólo tiene dos manos y diez dedos (...). El progreso vendrá por otro tipo de productividad, que es la productividad intelectual, pensar nuevas cosas, desartollo científico" (Col. Cam.). Sólo el 13,8\% manifiesta su acuerdo con la frase planteada y un $40 \%$ la matiza tal como decimos.

4. Creencia en una sociedad racional, pero no idilica. El conflicto se sigue manteniendo pero suavizado y superado a través del pacto social

Podemos ciftat en un $78,7 \%$ los que creen en una sociedad futura racional, aunque sólo el $22,5 \%$ de una forma utópica, sin ningún tipo de 
conflicto. Veremos más adelante la importancia cualitativa de este $22 \%$. Las objeciones se refieren básicamente al egoísmo y la irracionalidad def hombre. Un ejemplo claro: «Sólo entre comillas. Una forma racional sería la del hombre nuevo, éste que no existe ni creo vaya a existir, es una utopía absoluta. Como esto no es posible la sociedad nunca será racional porque el hombre no lo es, el animal y el instinto no desaparece ni desaparecerá nunca. Siempre habtá el poder, la ambición, la corrupción» (Técnico de empresa). Si es de aquí de donde vienen los problemas, no es de extrañat que nuestros entrevistados crean en la conciliación entre las difetentes clases (pregunta 11), aunque sea una conciliación parcial: el 32,5\% tesponde con un rotundo sí superador del conflicto, un $15 \%$ con un sí pero a través de la negociación, un $19,4 \%$ responde que no pero que son posibles los pactos, y un $16,9 \%$ que no, pero que el conficto se va atenuando. Hemos visto ya cómo se considera el desarrollo científico-técnico como instrumento de conciliación. Dos ejemplos más, significativos: «Pienso que se dan las condiciones tecnológicas, relativamente poco utópicas ya, para que existan los suficientes recursos... Si dedicamos éstos a temas científicos, de productividad y producción de nuevos bienes, obtendremos una sociedad abundante que, aunque tenga desequilibrios, lograría el consenso de la mayotía de la gente, siempre que esté organizada de una forma democtática y con una intervención del Estado que permita corregir esos desequilibrios» (Col. Econ.). «Es evidente, hay un nivel de diálogo mayor, sí que hay una conciliación en determinados aspectos, en gran parte porque la realidad se ha hecho más compleja: entre la minoría que tenía el poder (la propiedad de los medios de producción) y los trabajadores (que tenían sólo su fuerza de trabajo) ha aparecido dentro de la clase trabajadora, del conjunto de asalatiados, una serie de asalariados que tienen un papel clave en la marcha de las relaciones económicas y que de alguna manera representan una posibilidad de diálogo entre la propiedad, la organización del Estado y la capa superior de los trabajadores» (Col. Ing. Ind. y PSC).

El tipo de conciliación en que se cree queda clarificado en la pregunta 15 , cuando los entrevistados se ven fotzados a concretar un término. Sumando los que usan, sólo o con otro, cada uno de ellos, tenemos que un $48,7 \%$ cree en el pacto social, un 40,6 en la evolución y un $24,4 \%$ en la reforma. Ni uno solo de los entrevistados usa el término «revolución» (en el PSC se habla de «transformación» y en el PSUC de «mutación»). Todo parece indicat que se confía en un proceso objetivo, tecnológico y de progreso económico, que hace posible el pacto. Aunque un $13,1 \%$ es más optimista y nos habla de «cambio radical» o «revolución tecnológica». Un ejemplo de esta postura: «Más que una reforma, vamos a cambios importantísimos en la forma de vida, en el tiempo de trabajo y descanso, en el 
planteamiento global, en la organización de la sociedad, hacia la formación de superorganismos que son los únicos que tendrán las verdaderas soluciones a determinados problemas. Creo que hemos de buscar una palabra nueva. Nos podemos encontrar ante una sociedad postindustrial en muy poco tiempo, con unos problemas totalmente nuevos, con una relación capital-trabajo totalmente distinta. Pacto social creo que sí. Hemos de lle. gar a alguna forma de acuerdo social, plantearnos cómo los distintos grapos sociales encaran sus soluciones ante esta forma de vida nueva» (Círc. de Econ.).

\section{Consideración de la crisis actual como inicio de uria nueva era, en gran parte superadora de la alternativa o capitalismo o socialismo}

Ninguna pregunta hacía referencia explícita a este tema, pero sin embargo ha surgido una y otra vez en muchas de las respuestas. Como hemos visto, nuestros entrevistados creen en un progreso social paulatino, pero la confianza en el progreso científico-técnico y su papel determinante define la situación actual como momento clave. Esta idea es de hecho mayoritaria (definiendo en parte el tipo ideal $\mathrm{A}$, véase supra) pero además define, junto a otras respuestas, lo que he constatado como evanguardia de clase» ( $25 \%$ de los entrevistados): no sólo cambio tadical, sino salto a la utopía. Desgraciadamente, tengo que mantener el anonimato prometido, pero posiblemente la persona más conocida entre los entrevistados es buen ejemplo de esta vanguardia: «Estamos en un cambio de tal intensidad que ninguno de los principios que han configurado nuestra sociedad se mantiene (...). Evidentemente no será ni el socialismo ni el comunismo, ni nada de lo que hablamos en la sociedad actual (capitalismo incluido) sino, digan lo que digan, el humanismo que comportan las nuevas tecnologías (...).

"Creo que habrá unos hombres con capacidad de pensar, pero con una productividad tan grande con su pensamiento - gracias a las máquinas, entes nuevos creados por el hombre sin necesidad de vacaciones tit pagas dobles y sin visiones determinadas (justificadas) que atacan a la productividad- que tendrán que ser relativamente pocos los que piensen, hagan servir estas máquinas, frente a una gran masa de la población, no apta pata pensat, pero que tendrá que ser alimentada y culturizada, a su manera, por la sociedad. Ante ello el sindicalismo tiende a desaparecer, los partidos políticos también, e incluso el Estado tal como lo concebimos hoy. Vamos a asistir a la gran apoteosis de la sociedad civil, autoconstituyéndose, en la medida en que lo crea conveniente, porque los hombres con capa- 
cidad de pensar la vitalizan (...) estamos ante un futuro esplendoroso. En este sentido, el acuerdo social es ya hoy posible» (Círculo).

Desde otra óptica aparentemente distinta: «La humanidad avanza hacia una complejidad cada vez mayor, la cual obliga a una presencia de técnicos -especializados y de más bajo nivel- tanto en los aspectos de tecnología como de gestión. La sociedad se hace tan compleja que será cada vez más difícil decir cuáles son las clases dominantes si efectivamente el dominio es ya, en las sociedades occidentales, de quien detenta el capital: en definitiva, el último resorte del poder lo tiene el que puede aptetar el último botón, pero incluso éste está muy condicionado, mediatizado, por esta complejidad, por la necesaria presencia de una serie de profesionales imprescindibles para la marcha de la organización económica. Pienso que esta evolución llevará cada vez más a un nivel de relación más democrático, con mayor representación y presencia de los asalariados. Es de hecho la continuación de la evolución en marcha que lleva a la democracia como sistema político de relaciones imprescindible, y a este proceso de socialización de la propiedad de los medios de producción. Cada vez el accionista y el representante del capital contarán menos, el poder económico queda concentrado en el capital financiero y a escala internacional en las grandes multinacionales. El poder econónico ya no está cerca, sino cada vez más en manos de las grandes potencias y organizaciones, y ello se refleja a nivel político: lo que antes eran telaciones de poder a nivel de una nación, se van traduciendo en algo mucho más amplio. Peto esto es un proceso histórico hacia el socialismo. Lo que pasa es que el socialismo ya no es la utoṕ́a socialista, la sociedad sin clases (esto se ve cada día más lejos), sino esa sociedad compleja que se aleja del poder privado, que sustituye al empresario por el director, etc. La sociedad socialista es mucho más compleja, menos "limpia", de lo que pensaba la utopía" (Colegio de Ingenietos y PSC). «Estamos en lo que yo llamo una crisis época en Ia creación de algo distinto, un momento como la transición del esclavismo al feudalismo (...). La utopía existe, es una tealidad. Aunque no sea la teóricamente pensada por fulano o mengano - Marx - ni la parida por los países del Este. Es algo más real. No se pueden ideologizar los procesos» (PSUC).

\section{Defensa de la «tercera vía», de una «economía mixta»}

Lo hemos visto, en gran parte, en las respuestas ya seleccionadas, pero además nos queda claramente ratificado en las preguntas de la 6 a la 9 . En ellas se opta mayoritariamente por una economía de mercado, pero intervenida y planificada. El $38,7 \%$ habla de mercado con fuerte intervención 
estatal, el $24,4 \%$ de economía mixta y el 21,2 de plan que tenga en cuenta el mercado. La planificación $(88,8 \%)$ debe fijar «las reglas del juego», «el marco», "las coordenadas», lo que hemos tesumido en la expresión «condiciones generales de la producción»; pero de ninguna manera ha de ser una planificación autoritaria, totalmente centralizada. EI Estado lo que ha de hacer es (más allá de sus funciones de Estado del Bienestar, que lo es también) «hacer que el mercado funcione y cortegir sus imperfecciones». Podemos hablat, de hecho, de «mercado planificado», y ello no sólo en teoría, sino aplicado a la realidad española actual, en la que los entrevistados creen que es necesaria mayor intervención estatal, pero cualitativamente distinta, más eficaz, más planificadora y menos «bombero de empresas en dificultades».

Tres son las bases en que descansa la postura mayoritaria.

a) Defensa del mercado por su mayor eficiencia y estímulo: «En el fondo me pides que te confiese cuál es mi pensamiento sobre el modelo de sociedad. Prefero un tipo de sociedad en el que el metcado tenga unas funciones importantes. Básicamente, por una parte, en razón de la eficacia en la asignación y por otra la incentivación, la capacidad de iniciativa. Las funciones básicas han de ser las de destinar los recursos, básicamente en razón de su coste. Esto comporta una situación más tacional que una organización en que el Estado dirige totalmente la sociedad. (...) Aunque también es cierto que en el proceso de evolución histórica hay realmente mejoras en la vigilancia y el control de las distorsiones. Las sociedades posindustriales funcionan mejor que las de hace 70 o 100 años, y ello es consecuencia de la respuesta de la sociedad, del cambio en su organización a partir del incremento de las funciones del Estado y de la intervención de otro tipo de organismos paralelos: organizaciones de consumidores, sindicatos y otras organizaciones" (Generalitat y Círculo).

b) Pero el Estado y principalmente la planificación tepresenta el conocimiento, la "racionalización», a la par que hace posible el acuerdo social al corregir las injusticias del mercado: "Hace falta racionalización. E1 problema es hasta dónde. En algunos aspectos soy tremendamente intervencionista, aunque a veces me aterro porque llegaría a un intervencionismo tal que, ideológicamente, tengo que dat matcha attás. Me inclino por el intervencionismo buscando racionalidad pura, incluso llegando a imponer por la fuerza mayores desarrollos tecnológicos; incluso la cualificación: que los trabajos más importantes, más delicados, los hagan los más cualificados. La línea es que la intervención de los técnicos ha de ser preponderante» (Col. de Cam.). «La planificación democrática ayudaría mucho. ¿Qué es una planificación democtática? No los planes quinquenales de los páses socialistas, sino un estudio de la situación, la adopción de las 
líneas deducidas de la ciencia (...). Con una corrección constante a través de una gran participación social, de todos los elementos de la sociedad* (PSUC).

c) Se trata, sin embargo, de supetar la irracionalidad buroctática que significa la excesiva centralización. Ello se manifiesta en multitud de tespuestas diversas, valiendo la pena resaitar aquí las que hablan de auge de la sociedad civil. No son mayoritarias, peto sí las propias de la «vanguardia de clase», así como las que se atteven a usar el tétmino de «economía mixta»: «EI Estado tiene un papel en la economía moderna. Esto me patece más claro que el agua, dada la complejidad de aquélla y por tanto del mismo Estado. Me hace gracia cuando se dice que en EE UU no hay nacionalizaciones cuando existe la NASA: la mayor parte de las empresas trabajan para un cliente único que es el Estado. Peto lo que no tiene sentido es la distinción clásica mercado-Estado. Tomamos los dos extremos: la planificación central (que viene de Marx y principalmente de Lenin) y el mercado. En una sociedad moderna, ¿qué es una cosa y qué es la otra? Hemos de tener en cuenta además la Sociedad Civil. Si me hablas como ideal, creo que tendría que predominar la Sociedad Civil, que no el mercado, sobre el Estado. Ahora bien, este ideal se forma mediante compromisos (...). Al final, la burocracia privada y la pública se fusionan, pero el origen marca diferencias. En definitiva, la oposición mercado-Estado queda superada y lo importante es la distinción Estado - Sociedad Civil» (ESADE).

Particularmente significativas son las respuestas que muestran el acercamiento: «Eta clásico de las opiniones socialistas de hace años creer que se podía creat un sistema económico racional prescindiendo de las leyes del mercado. Creo que este es uno de los aspectos en que la doctrina socialista ha experimentado cambios. Se ha Ilegado a la convicción de que el mecanismo de metcado (no el mercado salvaje, naturalmente) es un factor necesario y beneficioso para la regulación económica y hasta cierto punto éste es el gran reto de las sociedades que se quieren acercar al socialismo (...). Dentro de las grandes líneas de la planificación, las leyes del mercado (sean sus agentes explotaciones individuales o públicas) pueden llenar un vacío que asegure la competitividad y el estímulo de la persona (...) un estímulo de superación que dé lugar a la realización personal» (PSC). «Es necesaria mayor intervención, aunque a mí no me gusta, para mí ya iba bien la época de la iniciativa privada, de la competencia, de la lucha entre unas empresas y otras. Pero ahora te das cuenta del inevitable cambio. Estamos, como dice Galbraith, en la "era de la incertidumbre", en una época de cambio mundial muy grande (...). Estamos saliendo de una era y a punto de entrar en otsa y todo se tambalea un poco (...). Es necesatia una mayor 
intervención del Estado tanto desde el punto de vista económico como político-social. El Estado juega además un papel central en la investigación y ello es básicos (Multin.).

Pensemos que las entrevistas fueron tealizadas en plena moda neoliberal. Sólo 2 de los entrevistados (Circulo y ESADE) reflejaron esta postura.

\section{Jerarquización profesional de la sociedad y dirección de ésta por la cúspide profesional}

Las respuestas a la pregunta 16 parecen mostrar una cierta división; por una parte, los profesionales han de tener un papel central y dirigente $(39,4 \%)$, pero por otra se han de limitar a ejercer como tales, cumplir exactamente su labor, «decidir los medios de acuerdo a unos fines que nos vienen dados» $(25 \%)$. El análisis cualitativo de las respuestas muestra que la dualidad es sólo aparente y se explica por la idea de jerarquización. Clatamente, las respuestas «cumplir como tales» lo que hacen es reivindicar la fuerza en que se basa el poder del profesional, su propiedad del sabet, su «neutralidad», el trabajo bien hecho que caracteriza teóricamente al profesional, mientras que las mayoritarias insisten en que la sociedad está basada ya en ese saber de forma que la función que corresponde al profesional es la de dirigente, principalmente al «buen profesional». Esta interpretación viene confirmada por las preguntas $21,17,18$ y 22 , que podemos considerar complementarias. Así, en la primera de ellas podemos constatat un $43,7 \%$ que considera frustrante, insatisfactoria, la función actual del profesional teivindicando claramente un proceso de tecnificación que se consideta aún insuficiente, mientras que un $22,5 \%$ responde de forma positiva a la pregunta, precisamente resaltando cómo ese proceso está ya en marcha (de nuevo la optimista «vanguardia de clase»). Un $15,6 \%$ exige mayor concreción y contesta «depende», dándonos posiblemente la clave de las dos posturas: «La situación de los profesionales varía mucho. Podríamos dividirios en dos grandes grupos: los que se han situado y los que acaban de entrar en la profesión. Los primeros desempeñan sus capacidades superando todos los síntomas de frustración, los segundos lógicamente tienen una situación en que dudan si utilizan todas sus capacidades a fordo» (Generalitat).

En cuanto a la satisfacción con el sistema educativo, es nula (ni una sola respuesta favorabie), contestando el $61,3 \%$ que es inadecuado a las necesidades del actual sistema productivo, y ello se concreta en una doble reivindicación que clarifica la idea de jerarquización defendida: se te- 
saita la necesidad de formación intermedia, de diferentes escalones que superen la simple separación estudios universitarios-ninguna cualificación profesional (aquí se incluye también una crítica a los estudios excesivamente teóricos), y necesidad de formación más amplia, altamente cualificada, de posgrado, para la cúspide directora (incluye $8,1 \%$ de «falta for. mación global»): "Tenemos un sistema malo, obsoleto y que no tesponde a los intereses de la sociedad. El modelo a seguir es el anglosajón, en el que la Universidad impulsa un tipo de formación que tiene que seguir la de. manda social a través de los distintos ciclos y a través de cursos superio. res» (Círculo).

Con todo, es en la pregunta 18 donde queda más clara la idea de jerarquización legitimada con la imagen de que se trata de una jeratquía de profesionales. Se juega con el término profesional entendido como propietario del conocimiento y también, cuando es necesario, como el que hace bien su trabajo. Así, determinados entrevistados hablan del portero, el mecánico, e incluso del basurero como profesionales que lo importante es que hagan bien su trabajo. El $53,1 \%$ cree en la superación de la división y el $35,6 \%$ la niega, pero tanto en un caso como otro con la idea de jerarquización, a través de la tecnificación del trabajo intelectual y mayor complejidad técnica del manual: «Se ha creado una clase intermedia, la tecnoestructura de Galbraith. A largo plazo el progreso tecnológico hará que buena parte de los manuales se incorporen a ésta, el gran núcleo del trabajo asalatiado será clase intelectual, tendrá un trabajo que implica cierta reflexión, pero por encima creo que subsistirán capas profesionales privilegiadas» (Generalitat).

La idea de jerarquización queda también patente, por último, en la diversidad de tespuestas a la pregunta 22 . Eil $36,9 \%$ sitúa a los profesionales como una nueva clase-jerarquía técnica, el $20,6 \%$ habla de asalaria. ción pero formando una élite diferenciada dentro del conjunto de trabajadores, y el $18,8 \%$ los considera un grupo intermedio entre el trabajo y el capital. Sólo un $5 \%$ los considera ligados al capital y el $18,8 \%$ tiene la idea de «nueva clase obrera» (profesionalizada-jerarquizada).

Creo que la siguiente respuesta ejemplifica la postura mayoritaria en el tema que nos ocupa: «La introducción del profesional en la jerarquía de la empresa o de la administración es totalmente positiva. El hecho de que los profesionales irtumpan a gestionar, Ia revolución de los managers, es

10. Es discutible la clasificación en el tipo $\mathrm{A}$ de una serie de militantes del PSUC que muestran más bien un marxismo ortodoxo. Los incluyo -aunque con la salvedad de esta nota- por no hacer un tipo específico (con 1 del PSC) y por su economicistoo-mecanicismo. 
totalmente positivo porque no forzosamente el capitalista o el político eran las personas más adecuadas para gestionar una empresa o una organización política. Creo firmemente en los profesionales como personas que han llevado a cabo los estudios necesarios para capacitarse en Ia línea de la actuación racional al frente de una empresa o de la Administración. El problema es que hoy en día aún no se da, hay un gran tesquicio entre las posibilidades y las necesidades» (Generalitat).

\section{Imporiancia de la diferenciación salarial basada en la jerarquia de conocimientos: a cada cual según su trabajo}

El tema es importante para nosotros dada nuestra tesis de que la nueva clase defiende un modelo de apropiación del conocimiento basado en la diferenciación salarial, y suficientemente ratificado. Ni una sola respuesta se muestra no partidaria de un abanico salarial, y el $46,2 \%$ cree que ya está bien el actual o que hay que ampliarlo. El $43,1 \%$ cree que la diferenciación ha de estar basada en la cualificación, la capacidad o el conocimiento, y el $36,2 \%$ en la responsabilidad; responsabilidad que tiene que ser retribuida, desde luego, según dicha capacidad: «Se ha de mantener un abanico salarial bastante abierto. Es decir, a un profesional se le ha de exigir mucho, pero se le ha de pagar. Un trabajador que tiene una faena manual, cuando deja el trabajo puede olvidarse de sus obligaciones en el trabajo, un profesional no (...) cuando sale tiene que seguir preocupado por el conjunto de cuestiones que envuelven la profesión» (PSC).

En la parte teórica de la tesis señalaba la máxima «a cada uno según su trabajo» como el ligamen de unión entre la nueva clase y la burocracia ubrera. De aquí la pregunta 20. El 61,2\% se muestra partidario de ella y sólo un 7,5\% la considera no posible. En gran parte, los pocos que la consideran como la máxima leninista igualitarista que es de origen .Para la mayotía se trata precisamente del funcionamiento del mercado, de la retribución a cada cual según lo que aporta, que es según su capacidad. En los partidos, lógicamente, está presente el contraste entre ambos significados y de aquí la importancia de sus tespuestas, que podemos resumir con ésta: «A mayor responsabilidad en la producción, mayor remunetación, y en este sentido los trabajos de dirección, superiores, tienen que estar más retribuidos. Creo que es evidente. E incluso aquí el caso del gerente de empresa, con una tetribución adecuada a su responsabilidad. El problema no es éste, son los beneficios del capital que no tienen ningún sentido» (PSUC).

En buena parte de los entrevistados se constatan planteamientos tatifica. dores de la desigualdad: «Indudablemente creo en el abanico salarial. Mis 
estudios no me los ha tegalado nadie $\mathrm{y}$ no puedo pensar que soy lo mismo que el señor que está conduciendo $\mathrm{el}$ autobús allá abajo, en la calle. No tiene que habet grandes desigualdades sociales, pero el esfuerzo se tiene que primar» (Generalitat). "Hay que mantener un amplio abanico salarial. Creo en unas grandes diferencias, que el esfuerzo y las ambiciones personales estén retribuidas, que sea un incentivo. Lo cual no excluye del todo la igualdad de oportunidades, pero sí un poco. La naturaleza es injusta de por sí. Evidentemente, el que nazca menos dotado tendrá menos oportunidades. Que tenga las mínimas, las dignas, pero no se puede esperat más. Las personas no nacen con las mismas oportunidades y nosotros no podemos hacer nada ante ello, es la única situación de injusticia, una injusticia natural, sin respuestas (Col. Ing. Ind.).

A resaltar que de las respuestas se deduce una cierta polémica entre el grado de aceptación de unos salarios para la cúspide muy por encima de la media. Desgraciadamente, no fue posible la medición cuantitativa de dicha aceptación. Con todo, su constatación, positiva o negativa, es una buena expresión de la idea de jerarquía.

\section{Conciencia y defensa, aunque moderadas, de la nueva clase}

Desde luego no es una idea comúnmente compartida, y de hecho casi sorptende el grado de aceptación de la nuteva clase por nuestros entrevistados, pero aun así ésta es moderada. Si la comparamos con los tipos ideales detectados, creo que podemos deducir que el grado de existencia de la nueva clase es superior a la autoconciencia que se tiene de ella. Con todo, ésta existe y entre una ligera mayoría. Ello lo vemos tanto en las preguntas que abordaban indirectamente el tema $(23,24)$ como en la última y directa. Muy alto me parece el porcentaje $-69,4 \%$ - de los que creen que existe convergencia entre los dos sistemas, máxime cuando las entrevistas fueron realizadas en pleno apogeo reaganiano y antes de la perestroika. Las razones de la convergencia las ve nuestra muestra en: a) se tiende a un «mercado sin propiedad», b) es un imperativo tecnológico y $c$ ) formación de una nueva clase dirigente común: «Lo que hay es una gran identidad desde el punto de vista de lo que llamaŕamos modelo industrial, es decir, técnicas de producción, productividades, tendimientos, utilización de tecnologías duras... Desde este punto de vista hay un gran parecido, y desde el punto de vista social también, en la medida en que tanto en el Este como en el Oeste hay una clase social emergente, la de los tecnócratas» (PSC). «Esta afirmación se ha planteado de dos maneras diferentes. Una ideológica, de superación de las ideologías y los sistemas. En 
ésta no creo. Peto sí en cambio en la otra, en la convergencia a partir de la formación de una nueva clase social dirigente común (...). Ambas generan su propio mecanismo de reconstrucción para seguir funcionando y este mecanismo es común: el mundo gerencial-burocrático» (ESADE).

Así, el $53,7 \%$ caracteriza la URSS por la formación de una nueva clase dirigente. Aunque no quedan muy claras las características de esta nueva clase. De este tipo de tespuestas el 31,3 habla de clase burocrática, el 29,2 de tecnoburocrática y un $39,5 \%$ no la define. A profundizar. Algunas respuestas a este nivel parecen mostrarnos que buena parte del no autorreconocimiento se debe a la mala prensa del término burocrático (y en parte tecnocrático). La nueva clase se quiete presentar como más pura: «Sí, se está formando una nueva clase, pero no estaría de acuerdo en llamatla tecnoburocrática. Se puede dar una organización basada en la técnica que no sea burocrática. No se puede confundir la organización a partir de la realidad técnica con la organización burocrática (...). Aunque realmente al hablar de nueva clase, al hablar Galbraith de tecnoestructura, decimos que la nueva clase detenta el poder, pero no toda organización del poder es buroctática (...). La base de la nueva clase es la técnica, desde la del Manager a la del Jefe de Taller, es una jerarquía, pero no burocrática, aunque sea una jetarquía de poder (...). Weber es poco conocido y normalmente se usa el término burocracia en un sentido negativo, con lo cual das a Ia nueva clase un sentido negativo que no tiene. Quizás sería mejor usar el término "gestores", traducción del de managers» (ESADE). Un buen resumen de la conciencia de clase.

También un $53,7 \%$ responde a la pregunta directa que sí existe la nueva clase en Occidente, aunque posiblemente el nivel de reconocimiento es mayor: otto $15 \%$ cree que existe pero sólo como "grupo social» sin format una clase, y un $16,3 \%$ señala que sí existe, pero que está al servicio del poder existente, sin poder propio. El $39,4 \%$ la considera positiva a ultranza, tacionalizadora.

Una última cita, complementaria de la anterior, como ejemplo de esta mitad de los entrevistados con conciencia de clase: "Creo mucho en esta nueva clase porque está alejada del capital y lejos de otros intereses que no son los más racionales. Está más preparada para ser racional que las otras (...). La tecnoestructura se sitúa en la línea del trabajador intelectual, que es cada vez más objetivo, ya que el conocimiento científco-técnico es mayor. El capital no ha de ser más que un instrumento que se aporta, pero el conjunto ha de ser llevado por la tecnoestructura, que es la que aporta la ciencia y la técnica a la dirección de las empresas. Creo que en la Administración también se está dando esta tecnoestructura. Concretamente en Catalunya, donde estrenamos Administración, esto es patente: 
los nuevos elementos ingresados pueden ser considerados como tecnoestructura» (Generalitat y Cítculo). Es todo un programa.

\section{GRUPO DE CONTRASTE}

Me he referido ya a éste. Realmente cubrió su función. El modelo es: tadicalmente distinto. Desgraciadamente no podemos profundizar en él, pero sí compararlo con los rasgos anteriores:

1. Concepción de la historia como lucha de clases. El $90 \%$ critica la idea de progreso.

2. Crítica al actual modelo de desarrollo científico-técnico. Igualmen. te el $90 \%$ habla de consecuencias negativas.

3. No confianza en el crecimiento, sino necesidad de lucha explícita contra la dominación. Sólo 1 respuesta opta por el crecimiento como objetivo, el $70 \%$ nos habla de la existencia de diferentes racionalidades y el $50 \%$ se manifiesta en contra de considerat la productividad palanca. clave.

4. No piensa en la superación del conflicto $(80 \%)$ y sólo un $10 \%$ cree en su atenuación.

5. Sí tiene en parte la idea de «nueva era» y de confluencia entre el capitalismo y el socialismo «real», pero en cuanto a nuevos métodos de dominación.

6. Defensa de una mayor intervenciónı del Estado, pero un Estado. basado en la democracia directa y una «auténtica autogestión» como alter. nativa al metcado y el plan.

7. Crítica a los profesionales y su modelo social $(90 \%)$.

8. «A cada uno según sus necesidades». Se explicita esta máxima, el $60 \%$ se declara contratio a la diferenciación salatial y el $30 \%$ cree que se ha de reducir al minimo.

9. Conciencia de la nueva clase, pero como nuevo modo de dominación. El $90 \%$ cree en la convergencia entre sistemas, el $50 \%$ define la URSS a través de la nueva clase, igualmente el $90 \%$ considera que existe la nueva clase en Occidente, para el $50 \%$ totalmente nueva y para el $40 \%$ como nueva forma del capital.

Los numerosos $90 \%$ se deben a que uno de los entrevistados sí podía integrarse en la misma ideología que nuestra muestra. Sirvió para refor* zar el contraste. 


\section{TIPOS IDEALES Y DIFERENCIAS ENTRE INSTITUCIONES}

Hasta aquí el análisis pregunta por pregunta. Es importante también el grado de coherencia de las distintas tespuestas entrevistado por entrevistado. Mediante un método complejo que no podemos resumir aquí, encontré a este nivel 4 tipos ideológicos ideales, a saber:

- Tipo A: Ideología explícitamente tecnoburocrática. Es el progreso científico-técnico el que solucionará los problemas sociales, de forma que los profesionales han de tener un papel dirigente.

- Tipo B: Ideología que podríamos llamar reformista. Aceptación de la dinámica actual, considerada como progreso y definida por una conciliación cada vez mayor de las clases en una sociedad progresivamente tacional. Este progreso es sin embatgo lento, paulatino, sin cambios brus$\cos$, ni siquiera como consecuencia del desarrollo científico-técnico, que es tan sólo un factor más del mismo.

- Tipo C: Conciencia de ctisis. Posturas tipo A o B, pero sobre las que predomina la sorpresa ante la crisis actual, considerada no sólo como económica, sino como crisis del modelo en que se creía, de forma que se muestran setias dudas sobre si el modelo seguitá siendo válido para el futuro.

- Tipo D: Ideología «escéptico-neutral». Las cosas son como son, no es tarea de los profesionales el cambiarlas, lo cual es además imposible. Se trata de irse adecuando lo mejor y más racionalmente posible a una dinámica imprevisible e independiente de nuestras voluntades.

La validez del grupo de contraste se ratifica con el hecho de que sólo uno de sus componentes puede ser incluido en uno de estos tipos, el tipo A en concreto, mientras que los otros formatian un quinto grupo $\mathrm{E}$, que tendriamos que definir como «tevolucionatio-autogestionario» o algo parecido.

Lo más significativo aquí será recoget los datos del número de entrevistados de cada tipo por instituciones: 
Conciencia tecnoburocrática en los grupos sociales dirigentes Partidos

\begin{tabular}{|c|c|c|c|c|c|c|c|}
\hline & & \multicolumn{2}{|c|}{ PSUC } & \multicolumn{2}{|c|}{ PSC } & \multicolumn{2}{|c|}{ Generalitat } \\
\hline & & Núm. & $\%$ & Núm. & $\%$ & Núm. & $\%$ \\
\hline Tipo A & $\ldots \ldots \ldots \ldots$ & 6 & 30 & 6 & 30 & 15 & 37,5 \\
\hline Tipo B & n........... & 11 & 55 & 8 & 40 & 14 & 35 \\
\hline Tipo C & n........... & 2 & 10 & -- & - & 1 & 2,5 \\
\hline Tipo D & $\ldots \ldots+\ldots$ & 1 & 5 & 6 & 30 & 10 & 25 \\
\hline Total & ............ & 20 & 100 & 20 & 100 & 40 & 100 \\
\hline
\end{tabular}

\section{Tecnoestructura}

\begin{tabular}{|c|c|c|c|c|c|c|}
\hline & \multicolumn{2}{|c|}{ Circulo } & \multicolumn{2}{|c|}{$E S A D E$} & \multicolumn{2}{|c|}{ Multinac. } \\
\hline & Núm. & $\%$ & Num. & $\%$ & Núm. & $\%$ \\
\hline Tipo A $\ldots \ldots \ldots \ldots . . .$. & 14 & 63,7 & 6 & 54,5 & 5 & 71,4 \\
\hline .............. & 6 & 27,3 & 3 & 27,3 & 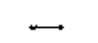 & - \\
\hline Tipo C .............. & 1 & 4,5 & 1 & 9,1 & - & - \\
\hline Tipo D & 1 & 4,5 & 1 & 9,1 & 2 & 28,6 \\
\hline Total ............. & 22 & 100 & 11 & 100 & 7 & 100 \\
\hline
\end{tabular}

Técnicos

\begin{tabular}{|c|c|c|c|c|c|c|c|c|}
\hline & \multicolumn{2}{|c|}{ Econom. } & \multicolumn{2}{|c|}{ Ing. Ind. } & \multicolumn{2}{|c|}{ Caminos } & \multicolumn{2}{|c|}{ Empresa } \\
\hline & Núm. & $\%$ & Núm. & $\%$ & Ním. & $\%$ & Núm. & $\%$ \\
\hline Tipo A. & 10 & 55,6 & 4 & 50 & 5 & 50 & 1 & 25 \\
\hline Tipo B .......... & 6 & 33,3 & 2 & 25 & 3 & 30 & 1 & 25 \\
\hline Tipo C .......... & - & - & - & 一 & - & - & - & - \\
\hline Tipo D ............ & 2 & 11,1 & 2 & 25 & 2 & 20 & 2 & 50 \\
\hline Total ......... & 18 & 100 & 8 & 100 & 10 & 100 & 4 & 100 \\
\hline
\end{tabular}


Cuadro Resumen

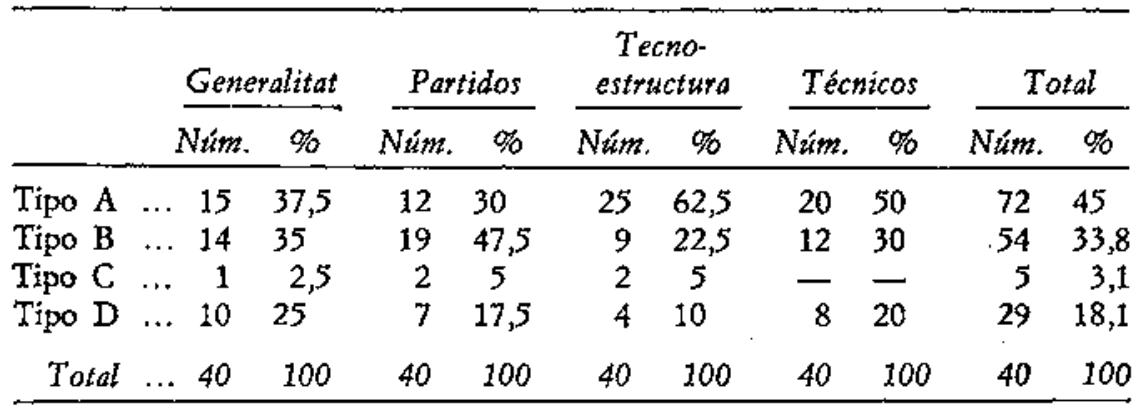

Predominio claro, más explícito de lo que yo pensaba en un principio, de la ideología propiamente tecnobutocrática. Existiría un cierto pudor ante el poder, aunque sea técnico, que en principio tenía que fomentar la postura tipo B. Con todo, voy a dejar las conclusiones para el lector. $\mathrm{Me}$ parece lo más adecuado en un artículo-resumen de este tipo. Tan sólo un pequeño comentario sobte la distribución de los diferentes tipos por instituciones. Es evidente que una de las riquezas mayores de la investigación realizada es la posibilidad de comparar la postura de cada una de éstas. Aquí tendremos que renunciar a ello, pero los datos anteriores nos pueden servir como consuelo, como visión muy general:

- Ya be señalado, y la nota 10 es importante en este sentido, que el PSUC es la frontera de nuestra muestra. Sus respuestas marcan una diferenciación-descontento con la situación actual- básica, pero, a mi entender, desde dentro (y el grupo de contraste es importante para verlo). Las tes* puestas a diversas preguntas dejan claro el mecanismo de integración del PSUC: la confianza en el progreso científico-técnico siempre positivo; en términos marxistas ortodoxos: la tendencia siempre creciente de las fuetzas productivas.

- Es lógico y a la vez ratificación de nuestra tesis de partida y de la selección de la muestra, el claro predominio del tipo A en la tecnoestructura. La cúspide es tecnoburocrática y es dentro de estas instituciones donde más clara se detecta la «vanguardia de clase». Con un comportamiento algo particular de ESADE a lo largo de todo el cuestionario: vanguardia de clase, pero autocrítica (aunque el carácter docente de buena parte de la muestra quizás ya da, de por sí, major rigor).

- A resaltar la concentración del tipo $\mathrm{D}$ en la Generalitat y los técnicos. ¿El no ascenso en la jerarquía hasta la misma cúspide produce un 
cierto escepticismo? Parece que se confirma, y no sólo en los datos aquí proporcionados, el PSC como expresión política de la jerarquía técnica...

Pero no nos dejemos llevar por la espectilación, lo importante hoy en el tema que nos ocupa es continuar la investigación concreta. A profundizar pues.

\section{ANEXO 1. CUESTIONARIO}

1. Edad.

2. Titulación.

3. Lugares y puestos de trabajo desde el final de los estudios profesionales.

4. En caso de que quiera manifestarlo, partico al que vota.

5. ¿Cree que la sociedad ha ido evolucionando a través de un continuo pro. greso? ¿Material y social?

6. ¿Cree posible la organización de la actividad económica de forma racional? ¿Cuál cree que han de ser las funciones del mercado y el Estado en esta organización tacional?

7. ¿Es posible concretar esta organización racional en unos objetivos económicos a definir? ¿Cuáles serían estos objetivos?

8. ¿Es la planificación económica el medio más adecuado para conseguir esos objetivos? ¿Qué tipo de planificación? Si la tespuesta es negativa, ¿cuál sería el medio idóneo?

9. ¿Cree necesaria una mayor intervención econớmica del Estado? ¿De qué tipo? ¿En España, hoy?

10. ¿Cree posible una organización racional de la sociedad? ¿La consecución de una futura sociedad racionalmente organizada, armónica, sin conflicto?

11. ¿Es posible la conciliación entre las diversas fuerzas sociales? Cómo?

12. ¿Cuál cree que puede ser el papel del desarrollo científico-técnico en esta conciliación de intereses y aquella organización racional? ¿Cuál cree que será el papel del progreso científico-técnico en la dinámica futura de la so. ciedad?

13. ¿Qué opina de los actuales movimientos ecologistas, de los Verdes alemanes, por ejemplo? ¿Cree necesaria una nueva tecnología? ¿X la energía nuclear?

14. ¿Qué opina de la frase «el incremento de la productividad es la palanca clave del progreso económico-social»?

15. ¿Con qué término definiría la dinámica social futura? Por ejemplo: ¿revolución?, ¿reforma?, ¿evolución?, ¿pacto social?, ¿otro término?

16. ¿Cuál cree que es el papel de los profesionales en esa organización racional de la que estamos hablando?

17. ¿Cree adecuado el actual sistema de cualificación profesional? ¿Y de los estudios universitatios? ¿Se forma adecuadamente a los profesionales para cumplir el papel que les corresponde? 
18. ¿Debe modificarse la actual división social del trabajo? ${ }_{\text {CCree conveniente }}$ la superación de la división trabajo intelectual-manual? ¿La cree posible en las próximas décadas?

19. ¿Cree necesatio un amplio abanico salarial o cree por el contrario que debe tendetse a una retribución más igualitatia?

20. ¿Que opina de la máxima «a cada uno según su trabajo»? ¿Cuáles cree que han de ser Ios criterios de diferenciacion salatial en el caso de que dicha diferenciación sea necesaria?

21. ¿Cree que la actual situación de los profesionales es Ia más adecuada al desempeño đe sus capacidades? Desde el punto de vista profesional, ¿e] proceso de superación del profesional que trabaja individualmente y su integxación en otganizaciones más amplias es positivo al disponer de más medios o negativo al anular su independencia o autonomía?

22. ¿Considera que se está produciendo una proletatización de los trabajadores intelectuales, tal como afirman algunos sociólogos? ¿Cuál cree que es la situación de los profesionales en la actual estructura de clases? ¿Más cercanos al trabajo o al capital?

23. ¿Existe una cierta convergencia económica y social entre EE.UU y la URSS, entre Occidente y la Europa Oriental, entre el capitalismo y el socialismo?

24. ¿Cómo calificaría los países del llamado «socialismo real», tealmente socialistas, de capitalismo de Estado, dominados por una «nueva clase» en la línea de Dijias; de alguna otra forma?

25. ¿Cree que se está produciendo en Occidente la formación de una nueva clase en la línea de Ia «tecnoestructura» definida por Gilbraith? En caso afirmativo, ¿qué intereses defendetía, con qué apoyos objetivos contaría? 